\title{
Privacy-Utility Tradeoff for Applications Using Energy Disaggregation of Smart-Meter Data
}

\author{
Mitsuhiro Hattori $^{1}{ }^{1}$ a) Takato Hirano $^{1}$ Nori Matsuda $^{1}$ Fumio Omatsu $^{1} \quad$ Rina Shimizu $^{1}$ \\ $\mathrm{YE} \mathrm{WANG}^{2}$
}

Received: November 27, 2017, Accepted: June 8, 2018

\begin{abstract}
Privacy-preserving data mining technologies have been studied extensively, and as one general approach, Calmon and Fawaz have proposed a data distortion mechanism based on a statistical inference attack framework. This theory has been extended by Erdogdu et al. to time-series data and been applied to energy disaggregation of smartmeter data. However, their theory assumes both smart-meter data and sensitive appliance state information are available when applying the privacy-preserving mechanism which is impractical in typical smart-meter systems where only the total power usage is available. In this paper, we extend their approach to enable the application of a privacy-utility tradeoff mechanism to such practical applications. Firstly, we define a system model which captures both the architecture of the smart-meter system and the practical constraints that the power usage of each appliance cannot be measured individually. This enables us to formalize the tradeoff problem more rigorously. Secondly, we propose a privacy-utility tradeoff mechanism for that system. We apply a linear Gaussian model assumption to the system and thereby reduce the problem of obtaining unobservable information to that of learning the system parameters. Finally, we conduct two experiments applying the proposed mechanism to the power usage data of actual households. The results of the two experiments show that the proposed mechanism works partly effectively; i.e., it prevents usage analysis of certain types of sensitive appliances while at the same time preserving that of non-sensitive appliances.
\end{abstract}

Keywords: privacy-preserving data mining, statistical inference, non-intrusive appliance load monitoring, convex optimization

\section{Introduction}

\subsection{Background}

The proliferation of personal devices capable of Internet connectivity has enabled new applications and services [3]. Examples include healthcare advice services based on the user's activity data captured by fitness tracking devices, navigation services based on GPS data from the user's smartphone, and demand response services based on power consumption data from household smart-meters. Such new services will definitely enrich our everyday lives.

At the same time, however, these services will collect users' personal data intentionally or unintentionally, which may in some cases violate their privacy [36]. In a well-known case, a retail company identified a teenage girl as pregnant based on her shopping habits [9], which can be thought of as illegal acquisition of sensitive information. The primary target of this paper is smartmeter data, which has been shown to potentially reveal the behavior of individuals [30], [31].

These privacy concerns in the era of the Internet of Things have triggered re-examination of privacy regulations around the world. For instance, the EU Parliament passed the General Data Protection Regulation (GDPR) in 2016. Most of the new privacy regula-

\footnotetext{
Mitsubishi Electric Corporation, Kamakura, Kanagawa 247-8501, Japan Mitsubishi Electric Research Laboratories, Cambridge, MA 02139, USA

Hattori.Mitsuhiro@eb.MitsubishiElectric.co.jp
}

tions including the GDPR now require explicitly that "natural persons should have control of their own personal data." ${ }^{* 1}$ It is therefore required that all service providers treat user's personal data carefully according to the demands of each individual. This social trend motivates the rapid development of privacy-preserving data mining technologies.

\subsection{Related Work}

A prominent line of privacy-preserving techniques is $k$ anonymity [35], [38] and its derivatives such as $\ell$-diversity [27], $t$-closeness [24] and $m$-invariance [40]. Their primary goal is to convert an aggregation of personal data into a non-personal (anonymous) dataset while preserving information as much as possible. Although their privacy metrics are intuitive and easy to evaluate, it is difficult or almost impossible to protect user privacy according to the detailed demands of each individual. Indeed, their basic strategy is to anonymize individuals by bundling similar records into indistinguishable bunches via generalization and omission of data, which in turn needs to ignore differences existing among individuals. Detailed demands of individuals, however, are intrinsically different since each individual has their own unique demands for privacy. Thus $k$-anonymity and its derivatives cannot fully take into account such detailed demands of individuals.

Differential privacy [10], [11] is in another line of research.

\footnotetext{
*1 In Recital 7 of the GDPR.
} 
Unlike $k$-anonymity and its derivatives, differential privacy defines the privacy metrics based on a rigorous mathematical framework. The privacy definition of differential privacy is such that an adversary querying the database, which contains personal data of many individuals, should find it difficult to determine whether the data record of any specific individual is even in the database. Accommodating users' specific privacy demands is therefore almost outside of their scope.

The work most relevant to ours is the consideration of privacy within a statistical inference attack framework [4], [8], [14], [15], [34]. In this framework, privacy is modeled as the amount of information obtained about the sensitive data when observing the released data. It is therefore possible to evaluate privacy on an individual basis by modeling the system with an appropriate definition of the sensitive and useful data. The primary goal of this framework is to find an optimal balance between the privacy of an individual and the utility of the service, and the problem of finding an optimal balance is formalized as an optimization problem where the objective function and constraint functions represent the privacy and utility. A solution of the optimization problem gives optimal privacy mapping which distorts the useful data to obtain privacy while still proving utility.

The theoretical aspect of this framework is proposed and analyzed by Calmon et al. [8] and extended by Basciftci et al. [4]. Salamatian et al. applied the theory to a Census dataset and TV rating dataset, and showed that it is indeed possible to reduce the revelation of political affiliation while enabling TV program recommendation services [34]. Erdogdu et al. extended the theory to time-series datasets and applied the extended theory to energy disaggregation of smart-meter data [14], [15]. They showed that it is possible to modify power data to conceal the usage of a sensitive appliance while still allowing detection of the usage of a useful appliance, where the useful and sensitive appliances in their experiments were respectively the washer-dryer and microwave.

Although Erdogdu et al. [14], [15] made a significant step towards applying the theory to real systems, there is still much room for improvement. For example, they considered only the case where both the smart-meter data and usage data of the sensitive appliances are directly observable. However, in case of actual use such as ordinary smart-meter systems, individual appliance usage data might not be directly observable. Therefore, it is desirable to achieve the optimal privacy mapping even in the case where usage of sensitive appliances is not available.

Privacy on smart-meter systems has been considered and tackled by many researchers [19], [25], [39], [41], [43]. Several of these privacy preserving techniques introduce additional batteries which are used to modify the power usage. However, introducing additional devices may be undesirable due to cost. Other solutions to the problem depend on a trusted third party [5], [12] or tamper-resistant devices [33] both of which will again raise the total cost of the system.

\subsection{Contribution}

In this paper, we extend the approach of Erdogdu et al. [14], [15] to apply a privacy-utility tradeoff mechanism to practical applications. As an example of practical applications, we consider

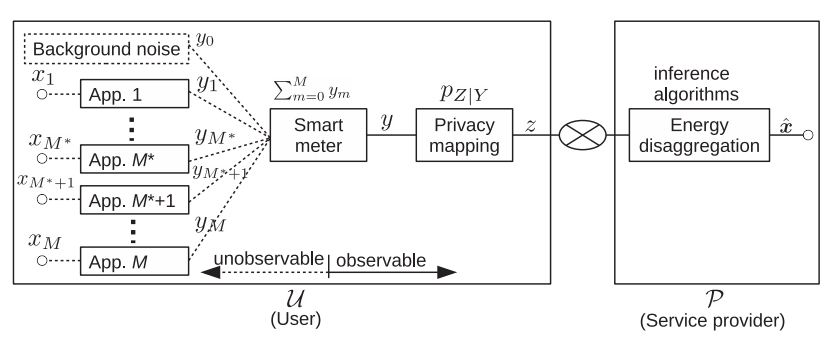

Fig. 1 Our system model. We assume App. 1 through App. $M^{*}$ are the appliances that a user $\mathcal{U}$ designated as sensitive, and App. $M^{*}+1$ through App. $M$ are those that $\mathcal{U}$ designated as non-sensitive. Solid lines represent observable data and dotted lines represent unobservable data.

in this paper an anomaly detection service for elderly residents living alone. The detection of anomalies with the residents can be based on the states of household appliances inferred via energy disaggregation. Using a smart-meter as a sensor device for anomaly detection is attractive because it requires no additional devices. This motivates many research efforts [1], [2], [37], but most of them pay little attention to privacy.

Firstly, we define a system model which captures both the architecture of the smart-meter system and the practical constraint that the power usage of each appliance cannot be measured individually. In our system model depicted in Fig. 1, modified smartmeter data is sent to a service provider who then conducts energy disaggregation of the smart-meter data to infer the appliance states. The energy disaggregation is conducted by a provider rather than on the user side since energy disaggregation often requires significant computational effort which may be impractical for the limited processing power of a smart-meter. The privacy issue here is that the provider may infer states of appliances that the user thinks of as sensitive. We capture this privacy issue by defining an adversary model and specifying the adversary's goal as well as his prior knowledge.

Secondly, we modify the optimization problem of Erdogdu et al. [14], [15] in such a way that individual appliance power usage data is not required. As we have noted, their privacy-utility tradeoff mechanism takes as input both the smart-meter data and usage data of the sensitive appliances. In our system model, however, the latter information is unavailable. We apply a linear Gaussian model to the system and thereby reduce the problem of determining an unknown system model to that of learning the model parameters such as the mean power consumption of each appliance and the hidden state distribution of the appliance states. These system parameters are, in some cases, available without conducting supervised learning on each household, because the mean power is often listed on a specification document of the appliance and the hidden state distribution can be simulated based on the typical usage pattern of the residents. Therefore, our mechanism is considered to be applicable to practical smart-meter systems.

Thirdly, we conduct two experiments applying the proposed mechanism to the power usage data of actual households. The first experiment uses a private dataset that we collected at one of the authors' household and the second experiment uses the public UK-DALE dataset [22]. For each experiment, we compute the system parameters that the adversary would possess a priori using supervised learning and compute the optimal privacy-utility 
tradeoffs for typical use cases. Then we distort the raw power data and evaluate the privacy and utility of the distorted data by examining the degradation of appliance usage inference performance. This paper quantitatively demonstrates that our mechanism is reasonable and effective, especially when high-power appliances such as the toaster are designated as sensitive.

\subsection{Organization of the Paper}

The rest of the paper is organized as follows. Section 2 elaborates our target application and defines a system model and an adversary model. Our theoretical analysis and proposition are given in Section 3, and experimental results and discussions are described in Section 4. Section 5 concludes the paper with future directions. Where necessary, detailed discussions are provided in the appendix.

\section{Target Application: System and Adversar- ial Models}

In this section, we first describe our target application and its privacy issue. Then we introduce notations used throughout the paper, and define a system model of the application and an adversary model of an "honest-but-curious" service provider.

\subsection{Target Application and Privacy Issue}

The target application we consider in this paper is an anomaly detection service for elderly residents living alone. More concretely, we consider an application where smart meter data, which is the aggregated power usage of all the appliances in a household, is collected from the house and disaggregated on a remote monitoring site, and appliance states are inferred whereby anomalies of the residents are detected. This service is proposed by Alcalá et al. [1], [2] and implemented by Song et al. [37].

The use of smart-meters for anomaly detection is preferable in that unlike anomaly detection using additional sensors such as wearable medical devices, we need no extra devices since smartmeters have already been installed in many countries and are ready for use. The rapid development of energy disaggregation technologies, also known as non-intrusive appliance load monitoring (NILM) [18], [21], [23], [28], [29], also motivates the use of smart-meters as sensor devices for anomaly detection.

A straightforward way of implementing this service would be to disaggregate and detect the anomaly state on the user side and notify it to the service provider. However, energy disaggregation and anomaly detection could be too computationally intensive to be performed efficiently in a typical smart-meter with limited processing and memory capabilities. Additionally, the performance of anomaly detection can be improved by comparing the smartmeter data of a user with that of other users, which is easily conducted on the provider side but difficult on the user side.

The privacy issue that we need to resolve in this application is that the service provider may infer states of the appliances that the user considers sensitive, as well as those required for anomaly detection. For example, the kettle is ideal for anomaly detection because many people, especially those in the UK, use it regularly and also think of it as a non-sensitive appliance. The hairdryer, on the other hand, is useful but many people (especially women) would think of it as sensitive because usage of the dryer implies that the user must have taken a bath. The difficulty of this issue lies in the fact that appliances differ across households and sensitivities also differ from person to person. It is therefore required to develop a privacy technology that can prevent the service provider from inferring states of the appliances that the user thinks of as sensitive while allowing inference of states of the non-sensitive appliances, based on the preference of each user.

From a cryptographic perspective, the service provider can be thought of as a so-called, "honest-but-curious" adversary, because the service provider basically obeys the protocol (providing anomaly detection service to the user) but at the same time tries to extract as much sensitive information as possible (inferring states of the appliances that the user thinks of as sensitive). We capture this adversarial situation with our adversary model in Section 2.3.2.

\subsection{Notation}

Suppose $X \in \mathcal{X}$ is a discrete random variable and $Y \in \mathcal{Y}$ is a continuous random variable, where $X$ and $\mathcal{Y}$ are some (possibly infinite) sets. We use capital $P_{X}(x)$ for the probability mass function of $X$ and small $p_{Y}(y)$ for the probability density function of $Y . E_{Y}[f(Y)]$ denotes the expected value of function $f(Y)$, i.e., $E_{Y}[f(Y)]=\int_{y} p_{Y}(y) f(y) d y$. We use $\mathcal{N}\left(\mu, \sigma^{2}\right)$ to denote the Gaussian distribution with mean $\mu$ and variance $\sigma^{2}$, and $p_{Y \mid X=x} \sim \mathcal{N}\left(\mu, \sigma^{2}\right)$ denotes that given that $X=x, Y$ is conditionally distributed according to the Gaussian distribution with mean $\mu$ and variance $\sigma^{2}$.

Let $\boldsymbol{X}=\left(\boldsymbol{X}^{*}, \overline{\boldsymbol{X}}\right)$ be a vector of discrete random variables representing the appliance states, where $\boldsymbol{X}^{*}=\left(X_{1}, X_{2}, \ldots, X_{M^{*}}\right)$ are discrete random variables of the sensitive appliance states and $\overline{\boldsymbol{X}}=\left(X_{M^{*}+1}, X_{M^{*}+2}, \ldots, X_{M}\right)$ are those of the non-sensitive appliance states, both of which are designated by $\mathcal{U}$.

\subsection{System and Adversary Models}

\subsubsection{System Model}

Our system model is depicted in Fig. 1.

Suppose there are $M$ appliances in the house of a user $\mathcal{U}$. Each appliance App. $m(m=1, \ldots, M)$ has several operating states denoted by $1, \ldots, K_{m}$, where $x_{m} \in\left\{1, \ldots, K_{m}\right\}$ denotes the realization of its operating state, and $y_{m}$ denotes its energy consumption. Note however that we cannot directly measure either $x_{m}$ or $y_{m}$, and can only measure the aggregated power usage $y=\sum_{m=0}^{M} y_{m}$ at the smart-meter, where $y_{0}$ is the background noise. The smartmeter data $y$ is then passed to the privacy mapping module which takes $y$ as input and maps it into the distorted data $z$. Here the mapping from $y$ to $z$ conforms to the conditional probability distribution $p_{Z \mid Y}$ which is computed beforehand by solving the privacy-utility tradeoff problem proposed in Section 3. The distorted data $z$ is then sent to a service provider $\mathcal{P}$, and $\mathcal{P}$ will conduct energy disaggregation with $z$ and infer the appliance states $\hat{\boldsymbol{x}}=\left(\hat{x}_{1}, \ldots, \hat{x}_{M}\right)$ using some inference algorithms.

The smart-meter measures the power usage $y$ regularly (typically every one minute), and the distorted data $z$ is sent to $\mathcal{P}$ successively. $\mathcal{P}$ may store all the time-series $z^{(1)}, \ldots, z^{(T)}$ for some time period $T$ (typically one month) and use them for inference 
of $\hat{\boldsymbol{x}}^{(1)}, \ldots, \hat{\boldsymbol{x}}^{(T)}$.

We should note here that although we modeled in Fig. 1 that the privacy mapping module is on the outside of the smartmeter, this is only for clarity and in practice it can be integrated into the smart-meter. Indeed, the privacy mapping operation is lightweight and can be executed with limited processing power and memory. However, in case precise power usage is required (for the sake of billing for example), another direct path from the smart-meter to the entity may exist. The smart-meter data $y$ will be sent to the entity through the path, possibly after aggregation, sampling or other privacy-preserving operation [26]. In our paper, we do not consider such a situation and focus only on the system model depicted in Fig. 1.

As mentioned above, the conditional probability distribution $p_{Z \mid Y}$ needs to be computed beforehand. This computation can be performed outside of the normal operation of the system by potentially either party, if computational resources permit. If computed by $\mathcal{P}, \mathcal{P}$ will be notified from $\mathcal{U}$ the model parameters such as the mean power consumption of each appliance and the hidden state distribution of appliance states, as well as the name of sensitive appliances. Some people might regard this notification to $\mathcal{P}$ as a privacy issue. However, we will leave this out of the scope of the process and focus only on the privacy issue arising from energy disaggregation; namely in this paper we will focus on sensitive appliance states which can be inferred from the smart-meter data. When deploying real systems, we need to tackle many issues such as who computes $p_{Z \mid Y}$, how $\mathcal{U}$ designates sensitive and non-sensitive appliances, how $\mathcal{P}$ obtains the model parameters and who determines a threshold value $\delta$ (appears in Section 3.1) and how. All of these should be addressed properly by taking into account the circumstances and constraints of the actual systems.

\subsubsection{Adversary Model}

The goal of an adversarial service provider $\mathcal{P}$ is to infer states of the appliances that $\mathcal{U}$ regards as sensitive. Suppose that $\mathcal{U}$ designated appliances App. 1 through App. $M^{*}$ as sensitive and App. $M^{*}+1$ through App. $M$ as non-sensitive. In this case, the adversarial goal of $\mathcal{P}$ is to infer $x_{1}, \ldots, x_{M^{*}}$ from $z$.

We assume that $\mathcal{P}$ knows all the appliances in $\mathcal{U}$ 's house. Also, we assume $\mathcal{P}$ knows the statistical distribution of each appliance.

The most typical probabilistic model used in energy disaggregation is the factorial hidden Markov model (FHMM) [16]. In FHMM, the emission distribution, transition probabilities and initial probabilities of all the appliances are used for inference of the hidden states. We therefore make the following specific assumptions. First, we assume that $\mathcal{P}$ knows App. $1, \ldots$, App. $M$, including the fact that $\mathcal{U}$ designated App. 1 through App. $M^{*}$ as sensitive and App. $M^{*}+1$ through App. $M$ as non-sensitive. $\mathcal{P}$ also knows the emission distribution $p_{Y_{m}^{(t)} \mid X_{m}^{(t)}}\left(y_{m} \mid x_{m}\right)$ for all $m=1, \ldots, M$ and $x_{m}=1, \ldots, K_{m}$, i.e., we assume that $\mathcal{P}$ knows the probability distribution of the power usage of App. $m$ at all the states $x_{m}$ for all $m$. $\mathcal{P}$ additionally knows the transition probabilities $P_{X_{m}^{(t+1)} \mid X_{m}^{(t)}}\left(x_{m}^{\prime} \mid x_{m}\right)$ and the initial probabilities $P_{X_{m}^{(1)}}\left(x_{m}\right)$ for all $m=1, \ldots, M$ and $x_{m}, x_{m}^{\prime}=1, \ldots, K_{m}$, i.e., the probability with which App. $m$ transits the state from $x_{m}$ to $x_{m}^{\prime}$ when the time steps from $t$ to $t+1$.

We now elaborate the justification of these assumptions.
In case of actual use, $\mathcal{P}$ does not necessarily need to know the parameters for the sensitive appliances App. 1, .., App. $M^{*}$. Namely, $\mathcal{P}$ does not need to know $p_{Y_{m}^{(t)} \mid X_{m}^{(t)}}\left(y_{m} \mid x_{m}\right)$ and $P_{X_{m}^{(t+1)} \mid X_{m}^{(t)}}\left(x_{m}^{\prime} \mid x_{m}\right)$ for $m=1, \ldots, M^{*}$. However, we make this assumption to consider a stronger adversary $\mathcal{P}$. This is because in general a security protocol is considered to be more secure if it is secure against a stronger adversary.

\section{A Proposed Privacy-Utility Tradeoff Mech- anism}

In this section, we modify the optimization problem of Erdogdu et al. [14], [15] in such a way that appliance usage data is not required. We formalize the optimization problem with definitions of privacy and utility in Section 3.1. We then show in Section 3.2 that the optimization problem is solvable, assuming that the joint probability distribution corresponding to the usage pattern of sensitive appliances and its contribution to the total power usage, which is unobservable in typical smart-meter systems, is known. These two subsections are merely a simple extension of the work of Erdogdu et al. [14], [15] to our system model. Our theoretical contribution appears in Section 3.3 where we modify the optimization problem so that we can interpolate the missing information by applying the linear Gaussian model assumption and thereby solve the problem.

Note that we define our optimization problem as a timeindependent one; namely, we optimize a data distortion mechanism that operates on each time-instant independently. We then apply it to sequential data, as shown in Section 4.

\subsection{Formalization of the Problem}

Here we formalize the privacy-utility tradeoff problem in a rigorous way.

\subsubsection{Definitions of Privacy and Utility}

The privacy metric we consider in this paper is as follows.

Definition 1 (Privacy metric). The privacy metric is the mutual information of sensitive appliance states $\boldsymbol{X}^{*}$ and distorted smartmeter data $Z$; i.e.,

$$
\begin{aligned}
& I\left(\boldsymbol{X}^{*} ; Z\right) \\
& \quad=\sum_{\boldsymbol{x}^{*} \in \boldsymbol{X}^{*}} P_{\boldsymbol{X}^{*}}\left(\boldsymbol{x}^{*}\right) \int_{Z} p_{Z \mid \boldsymbol{X}^{*}}\left(z \mid \boldsymbol{x}^{*}\right) \log \frac{p_{Z \mid \boldsymbol{X}^{*}}\left(z \mid \boldsymbol{x}^{*}\right)}{p_{Z}(z)} d z .
\end{aligned}
$$

The mutual information $I\left(X^{*} ; Z\right)$ represents the quantity of information one can obtain about $\boldsymbol{X}^{*}$ from the observed $Z$. It is therefore used extensively in the literature as a privacy metric [8], [14], [15], [32], [42]. Note however that $\boldsymbol{X}^{*}$ is a vector of discrete random variables while $Z$ is a continuous random variable, which is different from the situation considered in the literature where all the random variables were discrete. We therefore extended the theory.

Utility is measured by the following distortion metric.

Definition 2 (Distortion metric). Let $d: \mathcal{Y} \times \mathcal{Z} \rightarrow \mathbb{R}^{+}$be some distortion function ${ }^{* 2}$. The distortion metric is the expectation of $d(Y, Z)$; i.e.,

\footnotetext{
*2 Examples of distortion functions include the $L_{1}$ norm, $L_{2}$ norm and more
} generally $L_{p}$ norm. 


$$
E_{Y, Z}[d(Y, Z)]=\iint_{y \times Z} p_{Z \mid Y}(z \mid y) p_{Y}(y) d(y, z) d y d z
$$

Lower distortion intuitively corresponds to better utility.

However, the distortion metric in Definition 2 may appear slightly different from what we should deal with in this paper. Indeed, the ideal distortion metric would be the one that directly captures the degradation of the results of appliance usage analysis. However, the outcome of the appliance usage analysis depends heavily on the algorithms used for the analysis and therefore it is not practical to estimate the degradation in general. Also, empirically the distortion metric in Definition 2 is effective, as shown in Section 4.

\subsubsection{The Privacy-Utility Tradeoff Problem}

Suppose for now that the joint distribution $p_{X^{*}, Y}$ is already known. Then given $p_{X^{*}, Y}$, a distortion function $d$ and a distortion constraint $\delta$, the privacy mapping $p_{Z \mid Y}$ that minimizes the privacy information leakage can be found by solving the following optimization problem:

$$
\underset{p_{Z \mid Y}}{\arg \min } I\left(\boldsymbol{X}^{*} ; Z\right) \quad \text { subject to } E_{Y, Z}[d(Y, Z)] \leq \delta .
$$

\subsection{Computability and Convexity of the Optimization Prob- lem}

\subsubsection{Computability}

We show that both the objective function in Eq. (1) and constraint function in Eq. (2) can be computed respectively and therefore the optimization problem (3) is solvable, assuming $p_{\boldsymbol{X}^{*}, Y}$ is known.

We start with Eq. (1). First note that $X \rightarrow Y \rightarrow Z$ forms the Markov chain because the smart-meter data $Y$ depends on the appliance states $\boldsymbol{X}$ and the distorted data $Z$ depends on the smartmeter data $Y$. Then,

$$
p_{Z \mid X^{*}}\left(z \mid \boldsymbol{x}^{*}\right)=\int_{y} p_{Z \mid Y}(z \mid y) p_{Y \mid X^{*}}\left(y \mid \boldsymbol{x}^{*}\right) d y .
$$

Here,

$$
\begin{aligned}
& p_{Y \mid X^{*}}\left(y \mid \boldsymbol{x}^{*}\right)=\frac{p_{\boldsymbol{X}^{*}, Y}\left(\boldsymbol{x}^{*}, y\right)}{P_{\boldsymbol{X}^{*}}\left(\boldsymbol{x}^{*}\right)}, \\
& P_{\boldsymbol{X}^{*}}\left(\boldsymbol{x}^{*}\right)=\int_{y} p_{\boldsymbol{X}^{*}, Y}\left(\boldsymbol{x}^{*}, y\right) d y .
\end{aligned}
$$

Also,

$$
p_{Z}(z)=\sum_{\boldsymbol{x}^{*} \in \boldsymbol{X}^{*}} P_{\boldsymbol{X}^{*}}\left(\boldsymbol{x}^{*}\right) p_{Z \mid X^{*}}\left(z \mid \boldsymbol{x}^{*}\right) .
$$

Therefore, we can confirm that all the terms in Eq. (1) can be computed from $p_{\boldsymbol{X}^{*}, Y}$ and $p_{Z \mid Y}$.

As for Eq. (2), we can easily confirm the computability by seeing that

$$
p_{Y}(y)=\sum_{\boldsymbol{x}^{*} \in \mathcal{X}^{*}} p_{\boldsymbol{X}^{*}, Y}\left(\boldsymbol{x}^{*}, y\right) .
$$

\subsubsection{Convexity}

We additionally note here Eq. (3) is a convex optimization problem. This is because, as with Ref. [8], the objective function and the constraint function are convex functions of the optimization variable $p_{Z \mid Y}$.
Convex optimization has several desirable properties. From an analytical viewpoint, it is assured that any local minimum is a global minimum and finding a global minimum is therefore reduced to finding a local minimum [6]. From a practical viewpoint, efficient algorithms such as interior-point methods have been proposed, and software libraries are available Ref. [7].

\subsection{Gaussian Model Assumption}

We assumed in Section 3.1 that $p_{X^{*}, Y}$ is already known. In practical smart-meter systems, however, this assumption does not hold and we need to substitute $p_{\boldsymbol{X}^{*}, Y}$ with other known parameters ${ }^{* 3}$. We propose here the substitution method.

First, observe that from the law of total probability,

$$
\begin{aligned}
p_{\boldsymbol{X}^{*}, Y}\left(\boldsymbol{x}^{*}, y\right) & =\sum_{\overline{\boldsymbol{x}} \in \bar{X}} p_{\boldsymbol{X}^{*}, \overline{\boldsymbol{X}}, Y}\left(\boldsymbol{x}^{*}, \overline{\boldsymbol{x}}, y\right) \\
& =\sum_{\overline{\boldsymbol{x}} \in \overline{\boldsymbol{X}}} p_{\boldsymbol{X}, Y}(\boldsymbol{x}, y) \\
& =\sum_{\overline{\boldsymbol{x}} \in \overline{\boldsymbol{X}}} P_{\boldsymbol{X}}(\boldsymbol{x}) p_{Y \mid \boldsymbol{X}}(y \mid \boldsymbol{x}) .
\end{aligned}
$$

Now, computing $p_{\boldsymbol{X}^{*}, Y}\left(\boldsymbol{x}^{*}, y\right)$ boils down to computing $P_{\boldsymbol{X}}(\boldsymbol{x})$ and $p_{Y \mid X}(y \mid \boldsymbol{x})$.

In order to compute $p_{Y \mid X}(y \mid x)$, we apply a linear Gaussian model. This model has been used extensively to simulate the emission of home appliances in the energy disaggregation literature [17], [23], [28].

Let $Y_{0}$ be a random variable of the background noise and $Y_{m}$ be that of the emission of appliance $m$. Then,

$$
\begin{aligned}
& Y=Y_{0}+\sum_{m=1}^{M} Y_{m}, \\
& p_{Y_{0}} \sim \mathcal{N}\left(\mu_{0}, \sigma_{0}^{2}\right), \\
& p_{Y_{m} \mid X_{m}=x_{m, k}} \sim \mathcal{N}\left(\mu_{m, k}, \sigma_{m, k}^{2}\right),
\end{aligned}
$$

where $\mu_{0}$ and $\sigma_{0}^{2}$ are the mean and variance of the Gaussian distribution of the background noise, and $\mu_{m, k}$ and $\sigma_{m, k}^{2}$ are those of appliance $m$ in state $k$. Then, according to the standard probability theory [13],

$$
p_{Y \mid X=x} \sim \mathcal{N}\left(\mu_{0}+\sum_{m=1}^{M} \mu_{m, k}, \sigma_{0}^{2}+\sum_{m=1}^{M} \sigma_{m, k}^{2}\right) .
$$

Equation (13) implies that computing $p_{Y \mid X}$ is now reduced to obtaining the parameters $\Theta=\left\{\mu_{0}, \sigma_{0}^{2},\left\{\mu_{m, k}, \sigma_{m, k}^{2}\right\}\right.$. These parameters can be obtained either from the specification documents or reference models of the appliances, or by doing preliminary training activities.

Assuming that the variance of the total power data $Y$ is independent of the states of the appliances, which is merely for reducing model parameters in exchange for degradation of inference, Eq. (13) can further be simplified as

$$
p_{Y \mid X=x} \sim \mathcal{N}\left(\mu_{0}+\sum_{m=1}^{M} \mu_{m, k}, \sigma^{2}\right),
$$

*3 Note that we assume the adversary can obtain $p_{X^{*}, Y}$. This is for the sake of considering the strongest possible adversary. Refer to Section 2.3.2 for details. 
Table 1 Summary of the datasets used for the two experiments.

\begin{tabular}{|c|c|c|c|c|}
\hline \multicolumn{2}{|l|}{ Dataset } & \multirow{2}{*}{$\begin{array}{l}\text { Private dataset } \\
\text { (Experiment 1) }\end{array}$} & \multicolumn{2}{|c|}{ UK-DALE dataset } \\
\hline & & & Original & Used (Experiment 2) \\
\hline \multirow[t]{3}{*}{ Duration } & In total & $\begin{array}{c}\text { Dec. 26, } 2015 \text { - Jan. 3, } 2016 \\
\text { (9 days) }\end{array}$ & $\begin{array}{c}\text { Nov. 9, } 2012-\text { Apr. } 26,2017 \\
\text { (4.3 years) }\end{array}$ & $\begin{array}{c}\text { Aug. 1, } 2016-\text { Aug. 31, } 2016 \\
\text { (31 days) }\end{array}$ \\
\hline & For testing & Dec. 26 - Dec. 28 (3 days) & - & Aug. 1 - Aug. 7 (7 days) \\
\hline & For training & Dec. 29 - Jan. 3 (6 days) & - & Aug. 8 - Aug. 31 (24 days) \\
\hline Data type & Smart-meter & Power usage & \multicolumn{2}{|c|}{ Power usage } \\
\hline \multirow[t]{3}{*}{ Resolution } & Time & $1 / 60 \mathrm{~Hz}$ & $1 / 6 \mathrm{~Hz}$ & $1 / 60 \mathrm{~Hz}$ \\
\hline & Power usage & $7 \mathrm{~W}$ & \multicolumn{2}{|c|}{$1 \mathrm{~W}$} \\
\hline & Appliance states & Binary (ON/OFF) & - & Binary (ON/OFF) \\
\hline \multirow{2}{*}{$\begin{array}{l}\text { Number of } \\
\text { appliances }\end{array}$} & Existent & 18 & \multicolumn{2}{|c|}{53} \\
\hline & Used for experiments & 17 & - & 14 \\
\hline
\end{tabular}

where $\sigma^{2}$ is the variance of $Y$. In this case, computing $p_{Y \mid X}$ can be reduced to obtaining the parameters $\Theta^{\prime}=\left\{\mu_{0},\left\{\mu_{m, k}\right\}, \sigma^{2}\right\}$. We use this simplified model in Section 4.

$P_{X}(x)$ can also be obtained from the reference models of the appliances or by doing preliminary training activities.

Now, it is easy to see that $p_{\boldsymbol{X}^{*}, Y}$ can be obtained from $\Theta^{\prime}$ and $P_{X}$ and therefore the optimization problem is solvable.

\section{Experiments on Household Power Usage Data}

This section exhibits the results of our two experiments in applying the proposed mechanism to the power usage data of actual households. We give an overview of our experiments in Section 4.1 and explain the experimental procedure in Section 4.2. Sections 4.3 and 4.4 show the dataset, model parameters and results of Experiments 1 and 2, respectively. Section 4.5 discusses the implications of the results and effects of the assumptions made in the experiments.

\subsection{Overview}

Our goal of conducting two experiments is to examine whether the theory we propose in Section 3 is effective in an actual situation (i.e., in actual daily life) and is applicable to various households. To this end, we collected two different datasets: one is a private dataset which is used for Experiment 1 and the other is a public dataset which is used for Experiment 2. As for the private dataset, we designated one of the authors' house as a target household and recorded both the total power usage and appliance states of the household. As for the public dataset, we picked the UKDALE dataset [22] which contains fine-grained and long-period power usage of both the entire house and each individual appliance.

The two experiments were conducted as follows. First we collected and prepared the target dataset. We then applied a supervised learning algorithm to estimate the emission, transition and initial probabilities that we assume the adversarial $\mathcal{P}$ has full knowledge of. Then, we considered two use cases: 1) toaster is designated as sensitive; and 2) TV is designated as sensitive. For both cases, we tested many $\delta$ values, and for each $\delta$ we solved the convex optimization problem of Eq. (3) with $\Theta^{\prime}$ and $P_{X}$ and obtained privacy mapping $p_{Z \mid Y}$. We then distorted the power usage data according to $p_{Z \mid Y}$, and obtained the distorted power usage data. In order to evaluate the privacy and utility of our proposed mechanism, we applied an inference algorithm to both the raw and distorted data to infer the appliance usage of the sensitive and non-sensitive appliances, and compared the performance of the inference results.

Since the power usage data we used in our experiments are discrete values, we regard $Y$ and $Z$ as discrete random variables $\tilde{Y}$ and $\tilde{Z}$ respectively, and compute and apply a conditional probability mass function $P_{\tilde{Z} \mid \tilde{Y}}$. Discussion on the optimization problem with discrete random variables can be found in Appendix A.1.

\subsection{Procedure}

Both Experiments 1 and 2 are conducted according to the following four steps.

(1) Acquisition and preparation

(2) Computation of model parameters

(3) Optimization and distortion

(4) Evaluation of privacy and utility

We describe each of these steps below.

\subsubsection{Step 1: Acquisition and Preparation}

In this step, we acquire and prepare target datasets. Table $\mathbf{1}$ shows a summary of the datasets used for our experiments. Specific methods for the acquisition and preparation depend heavily on each dataset and details are deferred.

\subsubsection{Step 2: Estimation of Model Parameters}

In this step, we estimate the model parameters $\Theta^{\prime}$ and $P_{X}$ from the datasets. Since our adversary model given in Section 2.3.2 assumes that the adversarial $\mathcal{P}$ has full knowledge of the emission, transition and initial probabilities under the factorial hidden Markov model (FHMM), we apply a supervised learning algorithm to estimate these parameters. We do so by taking as input both the total power usage and the appliance states of the household as a ground truth, and estimating the hidden parameters by using the expectation-maximization algorithm.

For ease of estimation, though, we employ the following three assumptions.

Assumption 1: Each appliance is assumed to have only two possible states: ON and OFF.

Assumption 2: The appliance states are assumed to have converged to the steady-state, i.e., the initial state distributions 
are equal to the steady state distributions implied by the transition probabilities.

Assumption 3: The appliances are assumed to consume exactly $0 \mathrm{~W}$ when they are in the OFF state.

Assumptions 1 and 2 simplifies the estimation of $P_{X}$. Note that we have already assumed in Section 2.3.2 that all the appliances behave independently from each other, which is implied by employing FHMM as the underlying model. Hence we can estimate $P_{X}(x)$ as the product of the probabilities of each appliance; i.e., $P_{\boldsymbol{X}}(\boldsymbol{x})=\prod_{m=1}^{M} P_{X_{m}}\left(x_{m}\right)$. Assumption 1 reduces the number of transition and initial probabilities we need to estimate and Assumption 2 further eliminates the need of estimating the initial probabilities. Let $a_{m}$ be the transition probability of appliance $m$ from OFF to ON and $b_{m}$ be that of the opposite direction (ON to OFF). Then, according to Assumptions 1 and 2,

$$
P_{X_{m}}(\mathrm{ON})=\frac{a_{m}}{a_{m}+b_{m}}, \quad P_{X_{m}}(\mathrm{OFF})=\frac{b_{m}}{a_{m}+b_{m}} .
$$

Hence, $P_{X}$ can be easily estimated from only the ground truth of the appliance states.

Assumption 3 simplifies the estimation of $\Theta^{\prime}=\left\{\mu_{0},\left\{\mu_{m, k}\right\}, \sigma^{2}\right\}$. Indeed, Assumption 3 implies $\mu_{m \text {, OFF }}=0$ for all $m$ and therefore we only have to estimate $\Theta^{\prime}=\left\{\mu_{0},\left\{\mu_{m, \mathrm{ON}}\right\}, \sigma^{2}\right\}$ which can be estimated empirically.

The effects of these assumptions will be discussed in Section 4.5.

\subsubsection{Step 3: Optimization and Distortion}

In this step, we consider two of possible designation patterns of sensitive appliances, and for each pattern we solve the convex optimization problem and obtain a discrete privacy mapping $P_{\tilde{Z} \mid \tilde{Y}}$. Then, we distort the total power usage data according to the privacy mapping.

Concretely, We consider the following two designation patterns.

Pattern 1: Toaster is designated as sensitive

Pattern 2: TV is designated as sensitive

For each pattern, we solve the convex optimization problem, obtain a discrete privacy mapping and distort the total power usage data according to the privacy mapping. We repeat this process many times by changing the distortion constraint $\delta$.

We use the $L_{1}$ distance $d(\tilde{y}, \tilde{z})=|\tilde{y}-\tilde{z}|$ as the distortion metric. The optimization problems are solved by the convex optimization software $\mathrm{CVX}^{* 4}$.

\subsubsection{Step 4: Evaluation of Privacy and Utility}

In this step, we evaluate both the privacy and utility aspects of the distorted power usage data.

Since our goal of the privacy-utility tradeoff is to retain the inference of the non-sensitive appliance states while preventing that of the sensitive appliance states, we evaluate them by measuring the degradation of the appliance state inference. We therefore apply an inference algorithm to the raw and distorted power usage data to infer the hidden states of the appliances, and evaluate the performance of the inference.

We again model the hidden states using the FHMM with the parameters we obtained in Step 2, and infer the hidden states

*4 http://cvxr.com/cvx/

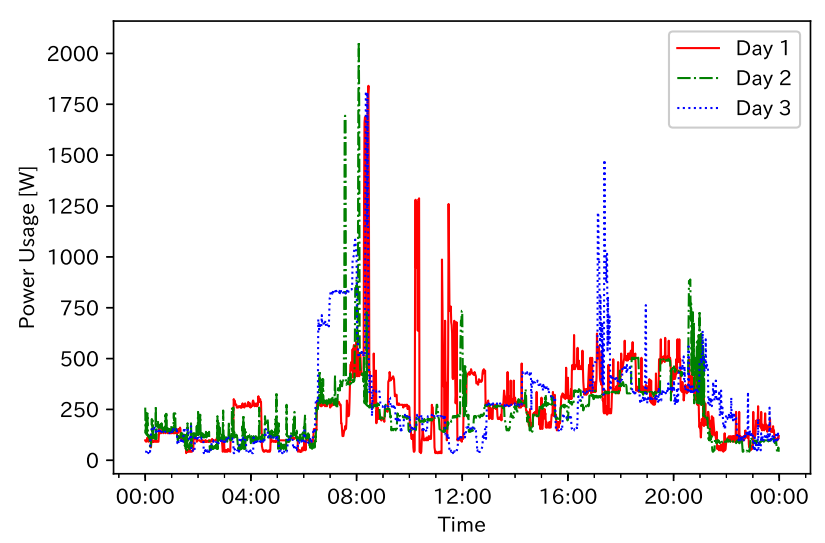

Fig. 2 Samples of the power usage data of Experiment 1.

using an approximate inference algorithm called the completely factorized variational approximation (CFVA) [16]. CFVA is used to avoid the computational complexity of exact inference algorithms. For this binary (ON and OFF) classification, the CFVA algorithm provides marginal posterior likelihoods which we can threshold at custom values to obtain a receiver operating characteristic (ROC) curve in order to evaluate the inference performance across different tradeoffs between true positive and false positive rates. We can also compute the area under the curve (AUC) which quantifies the inference performance across this tradeoff in a single number. We perform and compare this evaluation between the raw data and the distorted data.

In our target applications such as an anomaly detection service, we gain privacy (degradation of the AUC value of sensitive appliances) in exchange for loss of utility (degradation of the AUC values of non-sensitive appliances). Therefore, by comparing the AUC values of sensitive and non-sensitive appliances against various $\delta$, we can observe the privacy-utility tradeoff. Additionally we evaluate this tradeoff by changing the distortion constraint $\delta$ and plotting the AUC values of several appliances.

\subsection{Experiment 1 \\ 4.3.1 Dataset}

The dataset we used in Experiment 1 is a private dataset; we acquired the dataset by designating one author's home as a target household and by collecting the total power usage and appliance states of the target household. To collect the total power usage data, we attached to the circuit-breaker an electric power meter called the OWL+USB ${ }^{* 5}$ which records the electric power used in a household every minute. Due to limitations of the A/D converter used in the power meter, the resolution of the power recorded is $7 \mathrm{~W}$. We recorded the total power usage of the household for nine days. Samples are shown in Fig. 2 and the histogram is shown in Fig. 3. We also manually collected the ground truth of the appliance states for the same nine days. Table 2 shows an excerpt from the ground truth. A summary of the private dataset is shown in Table 1.

\subsubsection{Model Parameters}

We used six days of total power usage and appliance states data for the supervised learning, and computed the model parameters. In the supervised learning, we regarded the emission from the re-

\footnotetext{
*5 http://www.theowl.com/
} 


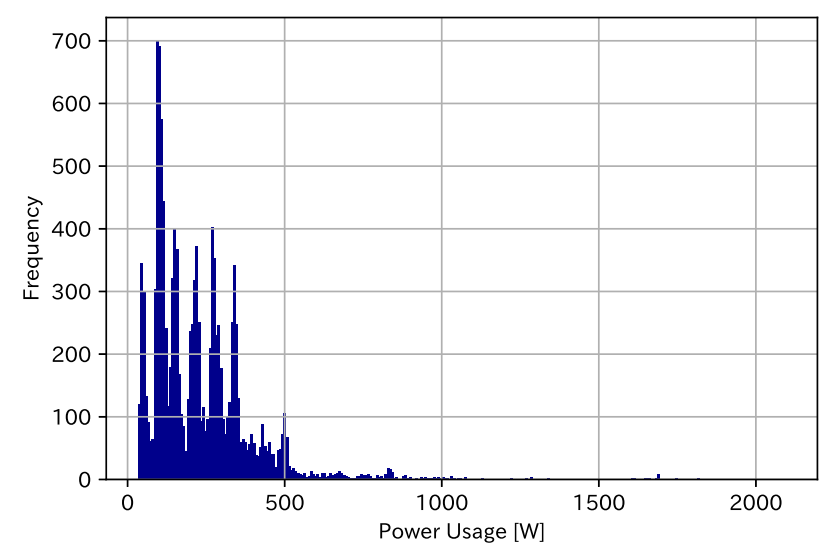

Fig. 3 Histogram of the power usage data of Experiment 1.

Table 2 An excerpt from the ground truth of the appliance usage of Experiment 1 .

\begin{tabular}{r|r|r|l|l}
\hline \hline Day & Time & $m$ & Appliance & Operation \\
\hline 1 & $3: 20$ & 11 & PC & ON \\
& $3: 20$ & 12 & Reading room light & ON \\
& $4: 16$ & 8 & Lavatory light & ON \\
& $4: 17$ & 8 & Lavatory light & OFF \\
& $\vdots$ & $\vdots$ & $\vdots$ & $\vdots$ \\
\hline 9 & $4: 33$ & 12 & Reading room light & ON \\
& $\vdots$ & $\vdots$ & $\vdots$ & $\vdots$ \\
& $23: 18$ & 9 & Living room light & OFF \\
& $23: 20$ & 14 & TV & OFF \\
\hline
\end{tabular}

Table 3 Appliances used in the target household of Experiment 1 and the parameters obtained from the supervised learning.

\begin{tabular}{r|l|r|l|l}
\hline \hline$m$ & Appliance & $\mu_{m, \mathrm{ON}}$ & $a_{m}$ & $b_{m}$ \\
\hline 0 & Background & & & \\
& + Refrigerator & 103.68 & & \\
1 & Bathroom light & 17.96 & 0.000491 & 0.0328 \\
2 & Electric heater & 391.91 & 0.000122 & 0.0208 \\
3 & Entrance light & 91.26 & 0.000484 & 0.222 \\
4 & Hairdryer & 413.22 & 0.000121 & 1.000 \\
5 & Kitchen light & 179.01 & 0.00234 & 0.0310 \\
6 & Kotatsu & 153.88 & 0.000623 & 0.0201 \\
7 & Laundry machine & 58.91 & 0.000500 & 0.0143 \\
8 & Lavatory light & 51.73 & 0.00365 & 0.517 \\
9 & Living room light & 83.85 & 0.00197 & 0.00548 \\
10 & Microwave & $1,099.66$ & 0.000121 & 0.500 \\
11 & PC & 113.15 & 0.000497 & 0.0181 \\
12 & Reading room light & 64.02 & 0.00175 & 0.0507 \\
13 & Rice steamer & 311.50 & 0.000244 & 0.0220 \\
14 & TV & 116.23 & 0.00323 & 0.00306 \\
15 & Toaster & $1,11.80$ & 0.000971 & 0.242 \\
16 & Vacuum cleaner & $1,214.21$ & 0.000121 & 1.000 \\
17 & Washstand light & 17.28 & 0.000605 & 0.333 \\
\hline \multicolumn{4}{|c}{$\sigma^{2}=5,440.4$} \\
\hline
\end{tabular}

frigerator as part of the background noise because it was always ON throughout the data collection. The results are shown in Table 3 .

\subsubsection{Optimization Results}

We solved the convex optimization problem and obtained a discrete privacy mapping $P_{\tilde{Z} \mid \tilde{Y}}$ for various $\delta$. Figure 4 shows a graphical representation of $P_{\tilde{Z} \mid \tilde{Y}}$ where the sensitive appliance is the toaster and $\delta=6$, and Fig. 5 shows that of $P_{\tilde{Z} \mid \tilde{Y}}$ where the sensitive appliance is the TV and $\delta=72$.

\subsubsection{Evaluation Results}

We inferred the appliance states from three days of total power usage data using CFVA, and evaluated the ROC curve and AUC

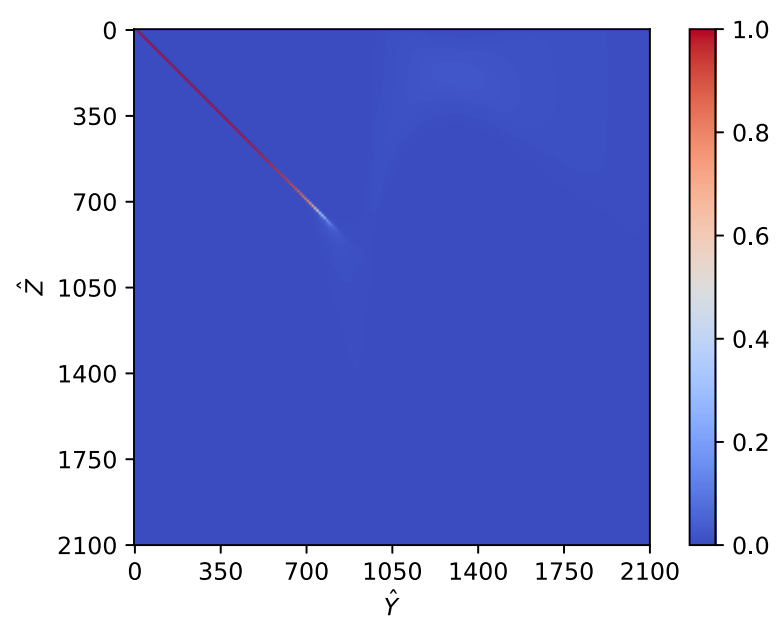

Fig. 4 A graphical representation of the privacy mapping $P_{\tilde{Z} \mid \tilde{Y}}$ (sensitive appliance is toaster and $\delta=6$ ).

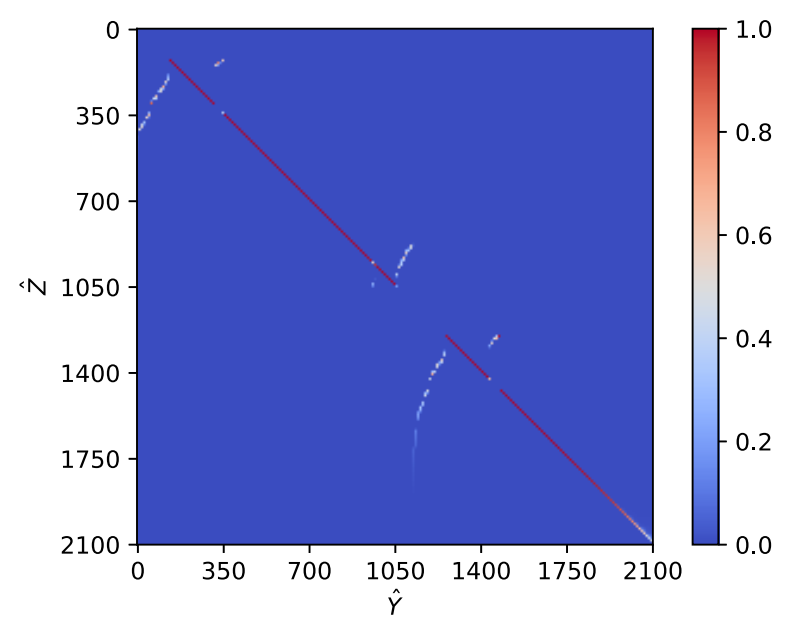

Fig. 5 A graphical representation of the privacy mapping $P_{\tilde{Z} \mid \tilde{Y}}$ (sensitive appliance is TV and $\delta=72$ ).

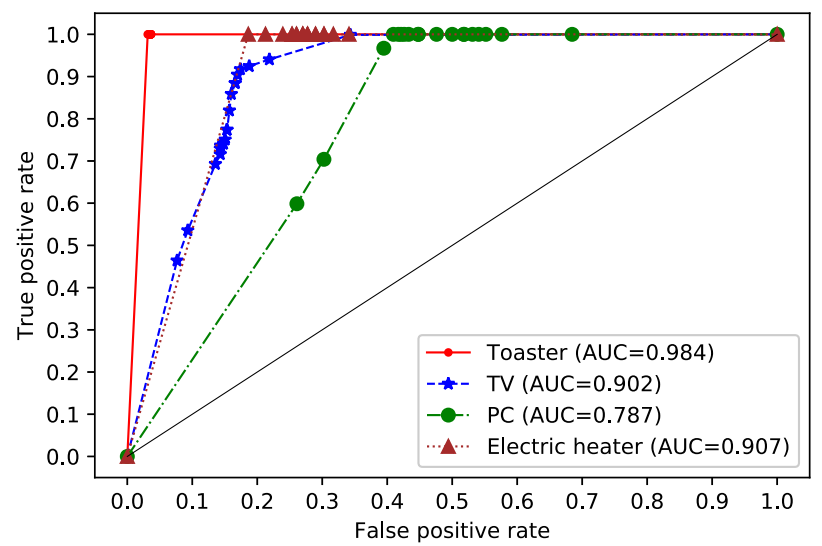

Fig. 6 ROC curves of the results of inference with raw data of Experiment 1. values.

Figure 6 shows the ROC curve and AUC values of the inference results of several appliances, where the analysis was performed on the raw data. As the AUC values show, the states of the toaster are inferred almost correctly, the states of the electric heater and TV are inferred with high accuracy, and the states of the $\mathrm{PC}$ are inferred with marginal accuracy.

Figure 7 gives ROC curves and AUC values of the inference results with the distorted data for Pattern 1. The inference performance for the toaster is severely degraded while the inference 


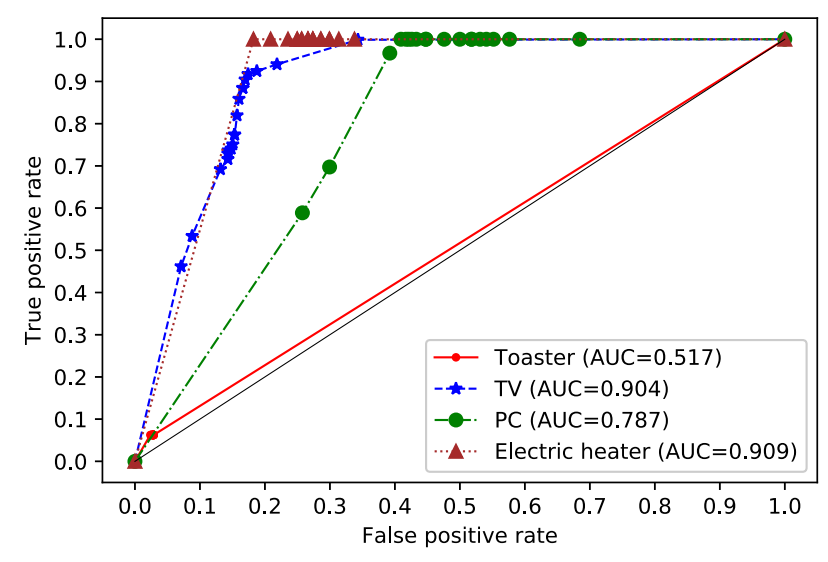

Fig. 7 ROC curves of the results of inference with distorted data of Experiment 1 (sensitive appliance is toaster and $\delta=6$ ).

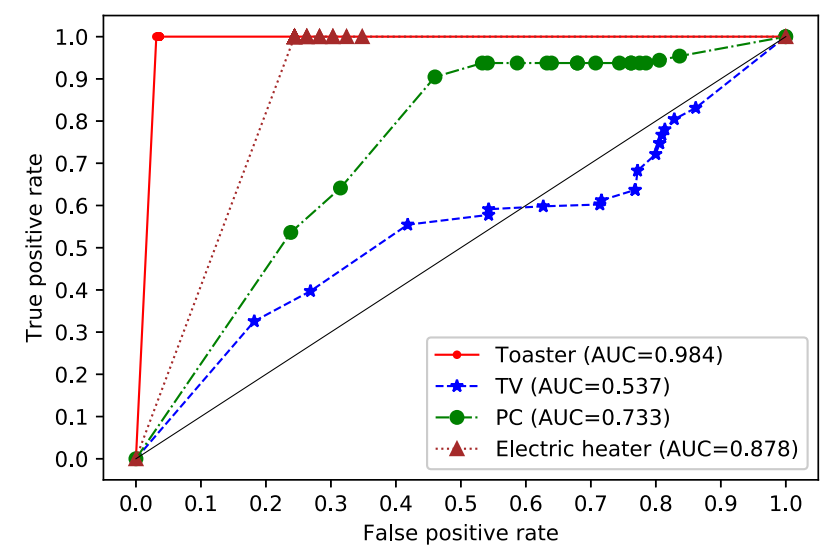

Fig. 8 ROC curves of the results of inference with distorted data of Experiment 1 (sensitive appliance is TV and $\delta=72$ ).

performance for the other appliances are preserved, as desired.

Figure 8 gives ROC curves and AUC values of the inference results with the distorted data for Pattern 2. The inference performance for the TV is degraded severely. The inference performance for the toaster and electric heater are preserved almost completely. The inference performance for the PC is degraded to some extent, but still enables meaningful inference.

Figure 9 shows the AUC values of the inference results with the distorted data for Pattern 1, where the distortion is performed using the privacy mapping computed from various $\delta$. Here $\delta$ is a distortion constraint as in Eq. (2), and a lower $\delta$ intuitively corresponds to better utility. An AUC value corresponds to the inference performance, where 1 implies appliance states are inferred perfectly and 0.5 implies the inference performance is equivalent to a random guess.

Figure 10 gives the AUC values of the inference results with the distorted data for Pattern 2.

\subsection{Experiment 2}

\subsubsection{Dataset}

The dataset we used in Experiment 2 is the UK-DALE dataset [22]. Details of the dataset including the data acquisition environment, data types and metadata types, and the statistics, are available in Refs. [20], [22].

The UK-DALE dataset contains data from five households with long periods of power usage records for each household. For our

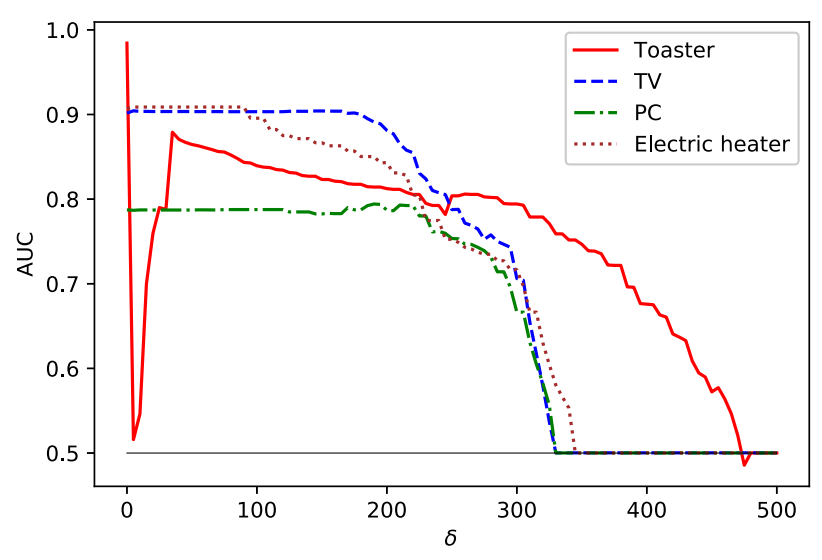

Fig. 9 AUC values of the results of inference with distorted data of Experiment 1 (sensitive appliance is toaster and distorted with various $\delta$ ).

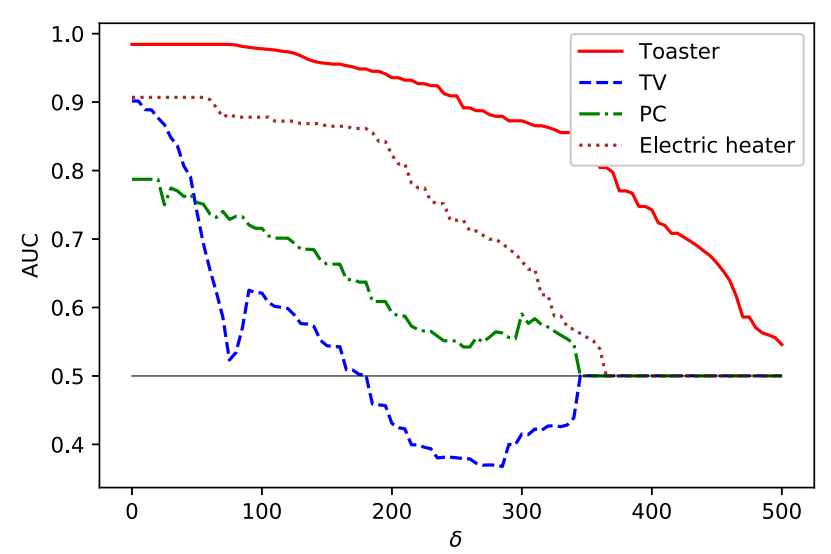

Fig. 10 AUC values of the results of inference with distorted data of Experiment 1 (sensitive appliance is TV and distorted with various $\delta$ ).

experiment, we used August 2016 data from House 1 because it has relatively low data loss. The House 1 data consists of data sampled at three different rates: $1 / 6 \mathrm{~Hz}, 1 \mathrm{~Hz}$, and $16 \mathrm{kHz}$, each of which contains the total power usage and appliance power usage data of the household that are captured according to the specified frequency. We used the $1 / 6 \mathrm{~Hz}$ data for our experiment.

The preparation process went even further. We downsampled the power usage from $1 / 6 \mathrm{~Hz}$ to $1 / 60 \mathrm{~Hz}$ so that it can be aligned with the private dataset we used in Experiment 1. Also, we modified the power usage data of individual appliances. The UKDALE dataset does not contain the ground truth of the appliance states. Instead it contains the power usage of individual appliances in the household ${ }^{* 6}$. We therefore converted the power usage of the appliances into the appliance states (ON and OFF) by referencing the threshold value of the appliances given in the metadata.

Samples of the total power usage are shown in Fig. 11 and the histogram is shown in Fig. 12. A summary of the original and UK-DALE dataset used in the experiments is shown in Table 1.

\subsubsection{Model Parameters}

We used 24 days of total power usage and appliance states data for the supervised learning, and computed the model parameters. For simplicity, though, we discarded those appliances whose estimated mean power is below $100 \mathrm{~W}$ and regarded them as a part

*6 To be precise, several appliances are accompanied by a binary operation data. However, they are not necessarily perfect and reliable, as stated in Ref. [20]. 


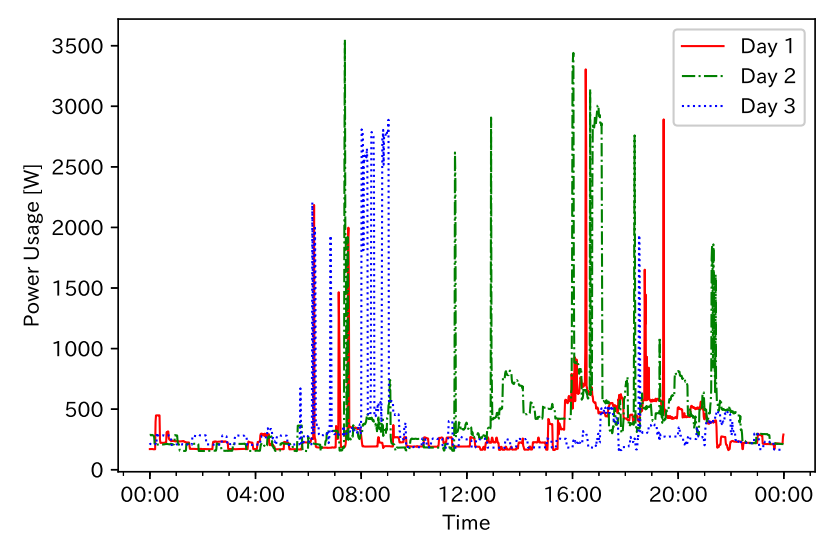

Fig. 11 Samples of the power usage data of Experiment 2.

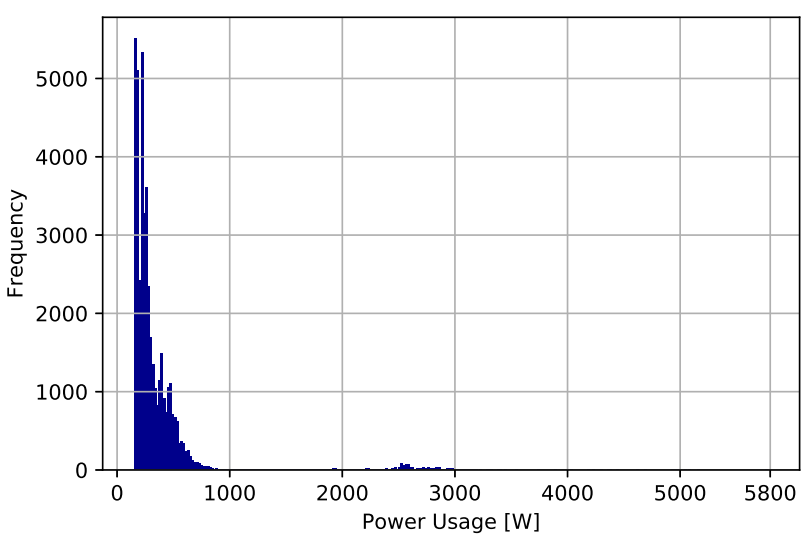

Fig. 12 Histogram of the power usage data of Experiment 2.

Table 4 Appliances used in the target household of Experiment 2 where the estimated mean power is greater than $100 \mathrm{~W}$ and their parameters were obtained from the supervised learning.

\begin{tabular}{r|l|r|l|l}
\hline \hline$m$ & Appliance & $\mu_{m, \mathrm{ON}}$ & $a_{m}$ & $b_{m}$ \\
\hline 0 & Background & & & \\
& + Small appliances & 244.92 & & \\
5 & Washing machine & 419.79 & 0.00141 & 0.0383 \\
6 & Dishwasher & 998.84 & 0.000868 & 0.0249 \\
7 & TV & 221.56 & 0.00141 & 0.00877 \\
8 & Kitchen lights & 132.06 & 0.00873 & 0.0501 \\
10 & Kettle & $2,292.28$ & 0.00247 & 0.680 \\
11 & Toaster & $1,623.87$ & 0.000928 & 0.376 \\
13 & Microwave & $1,435.61$ & 0.000406 & 0.226 \\
22 & Hoover & $1,744.13$ & 0.000464 & 0.162 \\
36 & Coffee machine & 197.37 & 0.000116 & 0.0208 \\
39 & Hair dryer & 785.21 & 0.000203 & 0.280 \\
40 & Straighteners & 206.15 & 0.000087 & 0.250 \\
44 & Child's table lamp & 309.20 & 0.000174 & 1.000 \\
49 & Office lamp2 & 115.35 & 0.000117 & 0.0135 \\
51 & PC & 347.33 & 0.000203 & 1.000 \\
\hline \multicolumn{4}{|c}{} & \multicolumn{3}{|c}{$\sigma^{2}=73,405.49$}
\end{tabular}

of the background noise. The results are shown in Table 4 .

\subsubsection{Optimization Results}

We solved the convex optimization problem and obtained a discrete privacy mapping $P_{\tilde{Z} \mid \tilde{Y}}$ for various $\delta$. Figure 13 shows a graphical representation of $P_{\tilde{Z} \mid \tilde{Y}}$ where the sensitive appliance is the toaster and $\delta=350$, and Fig. 14 shows a graphical representation of $P_{\tilde{Z} \mid \tilde{Y}}$ where the sensitive appliance is the TV and $\delta=625$.

\subsubsection{Evaluation Results}

We inferred the appliance states from seven days of total power usage data using CFVA, and evaluated the ROC curve and AUC values.

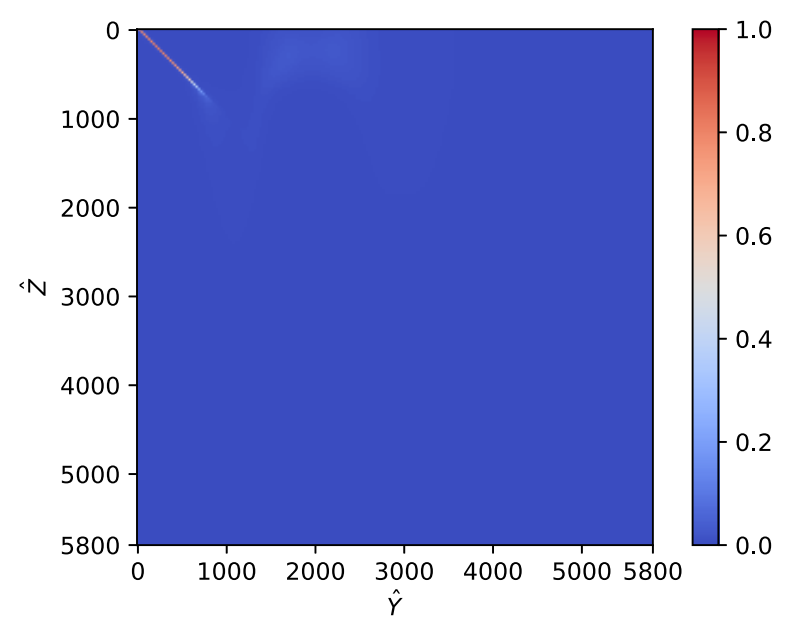

Fig. 13 A graphical representation of the privacy mapping $P_{\tilde{Z} \mid \tilde{Y}}$ (sensitive appliance is toaster and $\delta=350$ ).

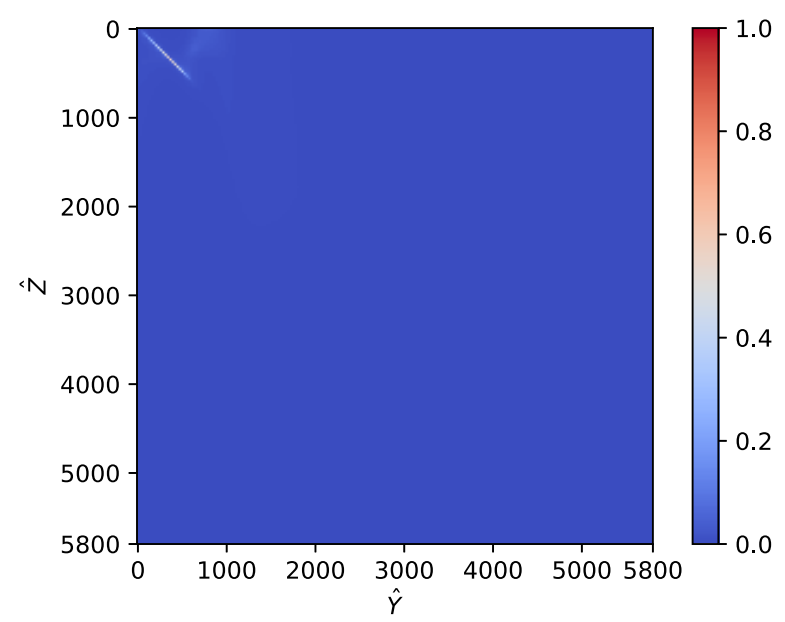

Fig. 14 A graphical representation of the privacy mapping $P_{\tilde{Z} \mid \tilde{Y}}$ (sensitive appliance is TV and $\delta=625$ ).

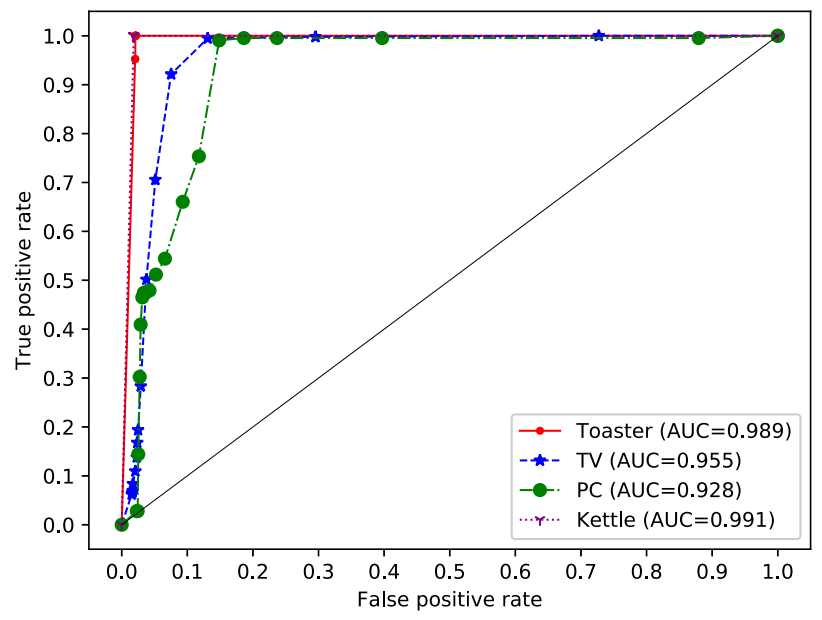

Fig. 15 ROC curves of the results of inference with raw data of Experiment 2 .

Figure 15 shows the ROC curve and AUC values of the inference results of several appliances, where the analysis was performed on the raw dataset. As the AUC values show, the states of the toaster and kettle are inferred almost correctly, the states of the TV are inferred with high accuracy, and the states of the PC are inferred with marginal accuracy.

Figure 16 gives ROC curves and AUC values of the inference 


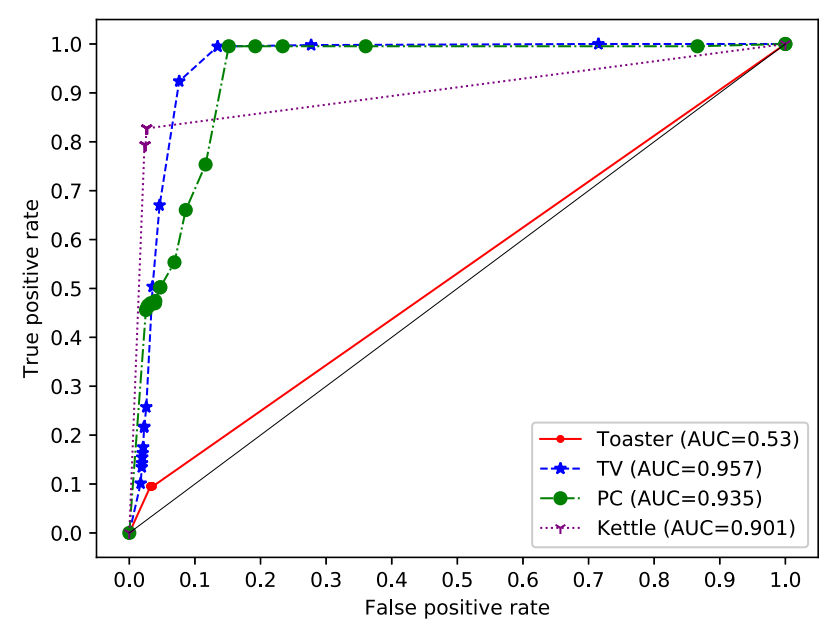

Fig. 16 ROC curves of the results of inference with distorted data of Experiment 2 (sensitive appliance is toaster and $\delta=350$ ).

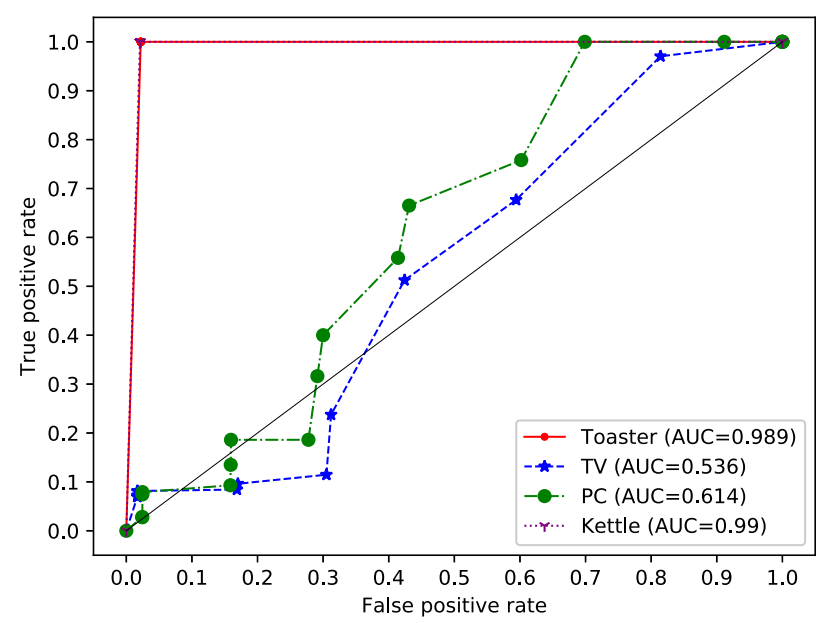

Fig. 17 ROC curves of the results of inference with distorted data of Experiment 2 (sensitive appliance is TV and $\delta=625$ ).

results with the distorted data for Pattern 1. The inference performance for the toaster is severely degraded while the inference performance for the other appliances are almost unchanged.

Figure 17 gives ROC curves and AUC values of the inference results with the distorted data for Pattern 2. The inference performance for the TV is degraded severely. The inference performance for the toaster and kettle are preserved. The inference performance for the PC is almost as much degraded as TV.

Figure 18 gives the AUC values of the inference results with the distorted data for Pattern 1.

Figure 19 gives the AUC values of the inference results with the distorted data for Pattern 2.

\subsection{Discussion}

As we have shown in Sections 4.3 and 4.4, the distortion works highly effectively for the case where the sensitive appliance is the toaster. This may be due to the fact that the toaster is realistically modeled with only two states: $\{\mathrm{ON}, \mathrm{OFF}\}$, and therefore our simplified model fits well. Moreover, the consumed power is as high as 1,000 W for Experiment 1 and 1,600 W for Experiment 2, which enables us to compute an optimal privacy mapping $P_{\tilde{Z} \mid \tilde{Y}}$ that attains both small mutual information and small distortion. Note that such high consumption rarely occurs as can be

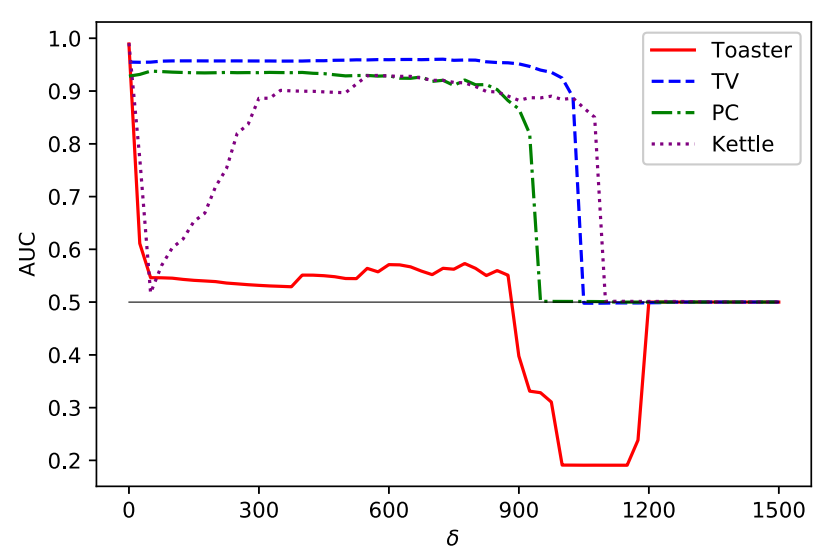

Fig. 18 AUC values of the results of inference with distorted data of Experiment 2 (sensitive appliance is toaster and distorted with various $\delta)$.

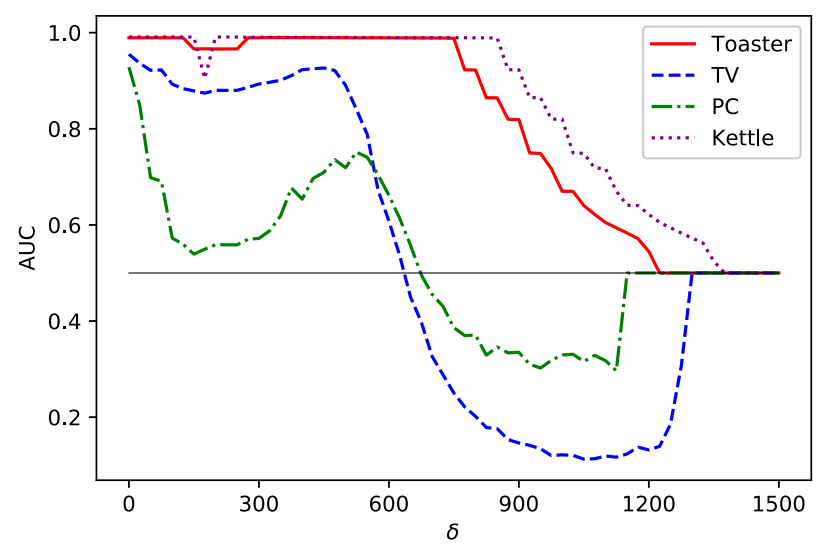

Fig. 19 AUC values of the results of inference with distorted data of Experiment 2 (sensitive appliance is TV and distorted with various $\delta$ ).

seen in Fig. 3 and Fig. 12, and thus the distortion of higher power values does not affect other low-power appliances.

On the other hand, for the case where the sensitive appliance is a television, the distortion not only renders inference of the sensitive appliance almost impossible but also degrades inference of the PC to some extent. This may stem from the fact that the TV consumes a relatively low power of $123 \mathrm{~W}$ for Experiment 1 and $232 \mathrm{~W}$ for Experiment 2 and thus distortion of middle power values would affect other middle-power appliances including the PC. Another possibility is that the PC takes not only two but multiple states and the distortion to the middle-range power usage degrades the inference.

It may be surprising that the inference algorithm could still detect to some extent the states of the PC, which has quite similar mean power parameters to the TV. However, as shown in Table 3, their transition probabilities are quite different, reflecting differences in frequency and duration of use. This difference possibly prevents the detection of the PC from suffering from the distortion of the power usage targeting the TV.

We now discuss the impact of the assumptions and approximations we made in the evaluation. We modeled the hidden states of the appliances with FHMM. FHMM is used typically in the energy disaggregation literature [23] and therefore this can be thought of as a reasonable modeling, but other inference algorithms such as neural networks [21] may give a greater advantage to an adversarial $\mathcal{P}$. We used a binary-state (ON and OFF) as- 
sumption for all the appliances. This may fit to some appliances (e.g., toaster) but not to others (e.g., PC). A model with more states will definitely give higher performance to both benign and adversarial $\mathcal{P}$. Use of an exact inference algorithm will make performance better at the price of computational complexity.

\section{Conclusion}

We propose in this paper a privacy-utility tradeoff mechanism which accommodates the situation where the sensitive appliance usage is not observable. We first describe a target application and its privacy issue, and give a system and adversary model. Then, we formalize the tradeoff as a convex optimization problem that we show can be solved. Finally, we exhibit experimental results on power usage data of actual households and show that the proposed mechanism is practical and effective.

Future work will be to extend this theory to the case where the service provider uses other inference algorithms such as neural networks.

Acknowledgments We would like to thank the anonymous referees for their valuable comments. We would like to thank Jack Kelly, William Knottenbelt and their anonymous colleagues for releasing their own power usage data. We would also like to thank all the volunteers including Chiyo, Kaori and Nagisa for their dedicated support of our experiment.

\section{References}

[1] Alcalá, J., Parson, O. and Rogers, A.: Detecting anomalies in activities of daily living of elderly residents via energy disaggregation and Cox processes, Proc. 2nd ACM International Conference on Embedded Systems for Energy-Efficient Built Environments: BuildSys '15, ACM Press (online), DOI: 10.1145/2821650.2821654 (2015).

[2] Alcalá, J., Urena, J. and Hernandez, A.: Activity supervision tool using non-intrusive load monitoring systems, 2015 IEEE 20th Conference on Emerging Technologies $\mathcal{E}$ Factory Automation (ETFA), IEEE (online), DOI: 10.1109/etfa.2015.7301622 (2015).

[3] Atzori, L., Iera, A. and Morabito, G.: The internet of things: A survey, Computer Networks, Vol.54, No.15, pp.2787-2805 (online), DOI: 10.1016/j.comnet.2010.05.010 (2010).

[4] Basciftci, Y.O., Wang, Y. and Ishwar, P.: On privacy-utility tradeoffs for constrained data release mechanisms, 2016 Information Theory and Applications Workshop (ITA), IEEE (online), DOI: 10.1109/ita.2016.7888175 (2016).

[5] Bohli, J.-M., Sorge, C. and Ugus, O.: A privacy model for smart metering, 2010 IEEE International Conference on Communications Workshops, IEEE (online), DOI: 10.1109/iccw.2010.5503916 (2010).

[6] Boyd, S. and Vandenberghe, L.: Convex optimization, Cambridge University Press (2004).

[7] Boyd, S., Vandenberghe, L. and Grant, M.: Advances in convex optimization, 2006 Chinese Control Conference, IEEE (online), DOI: 10. 1109/chicc.2006.280567 (2006).

[8] Calmon, F. and Fawaz, N.: Privacy against statistical inference, 2012 50th Annual Allerton Conference on Communication, Control, and Computing (Allerton), IEEE (online), DOI: 10.1109/allerton. 2012.6483382 (2012).

[9] Duhigg, C.: How companies learn your secrets, The New York Times, Vol.16, p.2012 (2012)

[10] Dwork, C.: Differential privacy, Automata, Languages and Programming, Springer Berlin Heidelberg, pp.1-12 (online), DOI: 10.1007/ 11787006_1 (2006).

[11] Dwork, C.: Differential privacy: A survey of results, Lecture Notes in Computer Science, Agrawal, M., Du, D., Duan, Z. and Li, A. (Eds.), Lecture Notes in Computer Science, Vol.4978, Springer Berlin Heidelberg, pp.1-19 (online), DOI: 10.1007/978-3-540-79228-4_1 (2008).

[12] Efthymiou, C. and Kalogridis, G.: Smart grid privacy via anonymization of smart metering data, 2010 1st IEEE International Conference on Smart Grid Communications, IEEE (online), DOI: 10.1109/ smartgrid.2010.5622050 (2010).

[13] Eisenberg, B. and Sullivan, R.: Why is the sum of independent nor- mal random variables normal?, Mathematics Magazine, Vol.81, No.5, pp.362-366 (2008).

[14] Erdogdu, M.A. and Fawaz, N.: Privacy-utility trade-off under continual observation, 2015 IEEE International Symposium on Information Theory (ISIT), pp.1801-1805, IEEE (online), DOI: 10.1109/isit.2015. 7282766 (2015).

[15] Erdogdu, M.A., Fawaz, N. and Montanari, A.: Privacy-utility tradeoff for time-series with application to smart-meter data, AAAI 2015 Workshop on Computational Sustainability (2015).

[16] Ghahramani, Z. and Jordan, M.I.: Factorial hidden Markov models, Machine Learning, Vol.29, No.2/3, pp.245-273 (1997).

[17] Guo, Z., Wang, Z.J. and Kashani, A.: Home appliance load modeling from aggregated smart meter data, IEEE Trans. Power Systems, Vol.30, No.1, pp.254-262 (online), DOI: 10.1109/tpwrs.2014. 2327041 (2015).

[18] Hart, G.: Nonintrusive appliance load monitoring, Proc. IEEE, Vol.80, No.12, pp.1870-1891 (online), DOI: 10.1109/5.192069 (1992).

[19] Kalogridis, G. and Denic, S.Z.: Data mining and privacy of personal behaviour types in smart grid, 2011 IEEE 11th International Conference on Data Mining Workshops, IEEE (online), DOI: 10.1109/icdmw.2011.58 (2011).

[20] Kelly, J.: Disaggregation of domestic smart meter energy data, $\mathrm{PhD}$ Thesis, University of London (2017).

[21] Kelly, J. and Knottenbelt, W.: Neural NILM: Deep neural networks applied to energy disaggregation, Proc. 2nd ACM International Conference on Embedded Systems for Energy-Efficient Built Environments: BuildSys '15, ACM Press (online), DOI: 10.1145/2821650. 2821672 (2015)

[22] Kelly, J. and Knottenbelt, W.: The UK-DALE dataset, domestic appliance-level electricity demand and whole-house demand from five UK homes, Scientific Data, Vol.2, 150007 (online), DOI: 10.1038/ sdata.2015.7 (2015).

[23] Kim, H., Marwah, M., Arlitt, M., Lyon, G. and Han, J.: Unsupervised disaggregation of low frequency power measurements, Proc. 2011 SIAM International Conference on Data Mining, pp.747-758, Society for Industrial and Applied Mathematics (online), DOI: 10.1137/1. 9781611972818.64 (2011).

[24] Li, N., Li, T. and Venkatasubramanian, S.: t-Closeness: privacy beyond k-anonymity and 1-diversity, 2007 IEEE 23rd International Conference on Data Engineering, pp.106-115, IEEE (online), DOI: 10 1109/icde.2007.367856 (2007).

[25] Li, S., Khisti, A. and Mahajan, A.: Privacy-optimal strategies for smart metering systems with a rechargeable battery, 2016 American Control Conference (ACC), IEEE (online), DOI: 10.1109/acc.2016.7525225 (2016).

[26] Lu, R.: Privacy-enhancing aggregation techniques for smart grid communications, Springer International Publishing (2016).

[27] Machanavajjhala, A., Kifer, D., Gehrke, J. and Venkitasubramaniam, M.: l-diversity: Privacy beyond k-anonymity, ACM Trans. Knowledge Discovery from Data, Vol.1, No.1, 3-es (online), DOI: 10.1145/ 1217299.1217302 (2007).

[28] Parson, O., Ghosh, S., Weal, M. and Rogers, A.: Non-intrusive load monitoring using prior models of general appliance types, AAAI 2012, pp.356-362 (2012).

[29] Parson, O., Ghosh, S., Weal, M. and Rogers, A.: An unsupervised training method for non-intrusive appliance load monitoring, Artificial Intelligence, Vol.217, pp.1-19 (online), DOI: 10.1016/j.artint. 2014.07.010 (2014).

[30] Pillitteri, V.Y. and Brewer, T.L.: Guidelines for smart grid cybersecurity, Internal Report NISTIR 7628 Revision 1, National Institute of Standards and Technology (2014).

[31] Quinn, E.L.: Smart metering and privacy: Existing laws and competing policies, SSRN Electronic Journal (2009).

[32] Rajagopalan, S.R., Sankar, L., Mohajer, S. and Poor, H.V.: Smart meter privacy: A utility-privacy framework, 2011 IEEE International Conference on Smart Grid Communications (SmartGridComm), IEEE (online), DOI: 10.1109/smartgridcomm.2011.6102315 (2011).

[33] Rial, A. and Danezis, G.: Privacy-preserving smart metering, Proc. 10th Annual ACM Workshop on Privacy in the Electronic Society WPES '11, ACM Press (online), DOI: 10.1145/2046556.2046564 (2011).

[34] Salamatian, S., Zhang, A., Calmon, F., Bhamidipati, S., Fawaz, N., Kveton, B., Oliveira, P. and Taft, N.: Managing Your private and public data: Bringing down inference attacks against your privacy, IEEE Journal of Selected Topics in Signal Processing, Vol.9, No.7, pp.12401255 (online), DOI: 10.1109/jstsp.2015.2442227 (2015).

[35] Samarati, P.: Protecting respondents identities in microdata release, IEEE Trans. Knowledge and Data Engineering, Vol.13, No.6, pp.1010-1027 (online), DOI: 10.1109/69.971193 (2001).

[36] Schneier, B.: Data and goliath: The hidden battles to collect your data and control your world, W.W. Norton \& Company (2015). 
[37] Song, H., Kalogridis, G. and Fan, Z.: Short paper: Time-dependent power load disaggregation with applications to daily activity monitoring, 2014 IEEE World Forum on Internet of Things (WF-IoT), IEEE (online), DOI: 10.1109/wf-iot.2014.6803150 (2014).

[38] Sweeney, L.: k-anonymity: A model for protecting privacy, International Journal of Uncertainty, Fuzziness and KnowledgeBased Systems, Vol.10, No.5, pp.557-570 (online), DOI: 10.1142/ s0218488502001648 (2002).

[39] Tan, O., Gunduz, D. and Poor, H.V.: Increasing smart meter privacy through energy harvesting and storage devices, IEEE Journal on Selected Areas in Communications, Vol.31, No.7, pp.1331-1341 (online), DOI: 10.1109/jsac.2013.130715 (2013).

[40] Xiao, X. and Tao, Y.: m-invariance: Towards privacy preserving republication of dynamic datasets, Proc. 2007 ACM SIGMOD International Conference on Management of Data: SIGMOD '07, pp.689700, ACM Press (online), DOI: 10.1145/1247480.1247556 (2007).

[41] Yang, L., Chen, X., Zhang, J. and Poor, H.V.: Cost-effective and privacy-preserving energy management for smart meters, IEEE Trans. Smart Grid, Vol.6, No.1, pp.486-495 (online), DOI: 10.1109/tsg. 2014.2343611 (2015).

[42] Yang, W., Li, N., Qi, Y., Qardaji, W., McLaughlin, S. and McDaniel, P.: Minimizing private data disclosures in the smart grid, Proc. 2012 ACM Conference on Computer and Communications Security: CCS '12, pp.415-427, ACM Press (online), DOI: 10.1145/2382196. 2382242 (2012).

[43] Yao, J. and Venkitasubramaniam, P.: The privacy analysis of battery control mechanisms in demand response: Revealing state approach and rate distortion bounds, IEEE Trans. Smart Grid, Vol.6, No.5, pp.2417-2425 (online), DOI: 10.1109/tsg.2015.2438035 (2015).

\section{Appendix}

\section{A.1 Modification from Continuous to Discrete Power Data}

In Section 3.3 we considered the case where the smart-meter data and distorted data are continuous. In practical situations, however, it is possible that the smart-meter data is quantized to discrete levels. Indeed, as we describe in detail in Section 4, we use discrete power data in Experiment 1 that has been quantized to a resolution of $7 \mathrm{~W}$. It is therefore required to modify the optimization problem Eq. (3) to accommodate such cases. We describe here the discretized version of the optimization problem.

Let $\tilde{Y} \in \tilde{Y}$ be a discrete random variable representing the quantized smart-meter data and $\tilde{Z} \in \tilde{Z}$ represent the distorted data, where $\tilde{\mathcal{Y}}$ and $\tilde{\mathcal{Z}}$ are finite sets. Let $d: \tilde{Y} \times \tilde{Z} \rightarrow \mathbb{R}^{+}$be some distortion function. Then the optimization problem Eq. (3) becomes

$\underset{p_{\tilde{Z} \mid \tilde{Y}}}{\arg \min } I\left(\boldsymbol{X}^{*} ; \tilde{Z}\right) \quad$ subject to $E_{\tilde{Y}, \tilde{Z}}[d(\tilde{Y}, \tilde{Z})] \leq \delta$

where

$$
\begin{aligned}
& I\left(\boldsymbol{X}^{*} ; \tilde{Z}\right) \\
& =\sum_{\boldsymbol{x}^{*} \in \mathcal{X}^{*}} \sum_{\tilde{z} \in \tilde{Z}} P_{\mathcal{X}}\left(\boldsymbol{x}^{*}\right) P_{\tilde{Z} \mid X^{*}}\left(\tilde{z} \mid x^{*}\right) \log \frac{P_{\tilde{Z} \mid X^{*}}\left(\tilde{z} \mid x^{*}\right)}{P_{\tilde{Z}}(\tilde{z})}, \\
& E_{\tilde{Y}, \tilde{Z}}[d(\tilde{Y}, \tilde{Z})]=\sum_{\tilde{y} \in \tilde{y}} \sum_{\tilde{z} \in \tilde{Z}} P_{\tilde{Z} \mid \tilde{Y}}(\tilde{z} \mid \tilde{y}) P_{\tilde{Y}}(\tilde{y}) d(\tilde{y}, \tilde{z}) .
\end{aligned}
$$

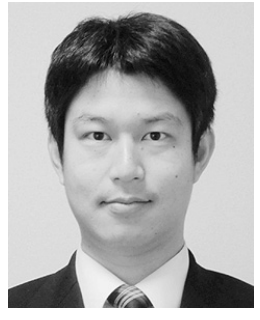

Mitsuhiro Hattori received his B.E. and M.E. degrees from Kyoto University, Japan, in 2003 and 2005, respectively. He joined Mitsubishi Electric Corporation in 2005, and since then he has been engaged in the research and development of information security. He is a member of IEICE and IPSJ.

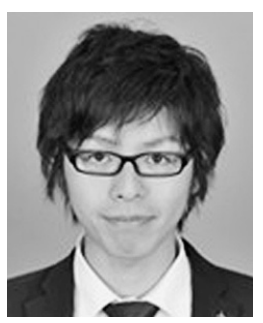

Takato Hirano received his B.A. degree from Osaka Kyoiku University in 2004, his M.S. degree from Tokyo Metropolitan University in 2006, and his D.S. degree from Tokyo Institute of Technology in 2010. He joined Mitsubishi Electric Corporation in 2010, and since then he has been engaged in the research and development of information security. He is a member of IEICE and IPSJ.

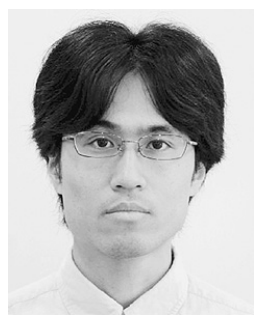

Nori Matsuda received his B.E. and M.E. degrees from Chuo University, Japan, in 1995 and 1997, respectively. He joined Mitsubishi Electric Corporation in 1997, and since then he has been engaged in the research and development of information security. He is a member of IEICE.

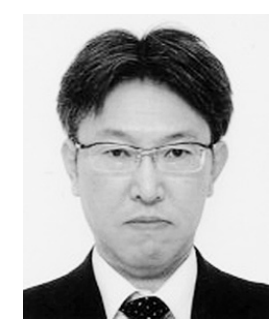

Fumio Omatsu received his B.E. degree from Tokyo Polytechnic University, Japan, in 1990. He joined Mitsubishi Electric Corporation in 1990, and since then he has been engaged in the research and development of information network management and information security. He is a member of IPSJ.

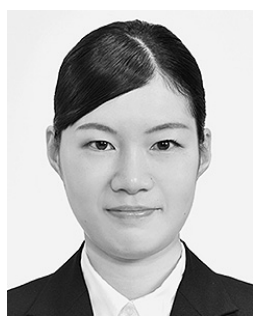

Rina Shimizu received her B.S. degree from Nara Women's University in 2013 and her M.E. degree from Kyoto University in 2015. She joined Mitsubishi Electric Corporation in 2015, and since then she has been engaged in the research and development of information security. 


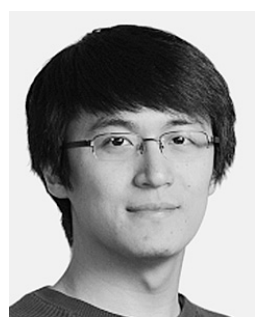

Ye Wang received his B.S. degree from Worcester Polytechnic Institute in 2005, and his M.S. and Ph.D. degrees from Boston University in 2009 and 2011. He joined Mitsubishi Electric Research Laboratories in 2012, and since then he has been engaged in the research and development of information security, signal processing, machine learning, and communications. 